\title{
À la recherche des traces du galicien dans le catalan
}

Exilés politiques ou émigrés économiques, les migrants comme « lieu du contact » des langues

\section{Emma Rubio-Milet}

\section{(2) OpenEdition}

\section{Journals}

Édition électronique

URL : http://journals.openedition.org/agedor/4767

DOI : 10.4000 /agedor.4767

ISSN : 2104-3353

Éditeur

Laboratoire LISAA

Référence électronique

Emma Rubio-Milet, « À la recherche des traces du galicien dans le catalan », L'Âge d'or [En ligne], 12 I

2019, mis en ligne le 01 octobre 2020, consulté le 15 octobre 2020. URL : http://

journals.openedition.org/agedor/4767 ; DOI : https://doi.org/10.4000/agedor.4767

Ce document a été généré automatiquement le 15 octobre 2020.

L'Âge d'or. Images dans le monde ibérique et ibéricoaméricain 


\section{À la recherche des traces du galicien dans le catalan}

Exilés politiques ou émigrés économiques, les migrants comme « lieu du contact » des langues

\section{Emma Rubio-Milet}

1 Pour les migrants, la question linguistique est au cœur des nombreuses difficultés, contraintes et injonctions d'adaptation qu'ils doivent affronter au cours de leur(s) déplacement(s). Très rarement les migrations se produisent entre pays où l'on parle la même langue. Le monde hispanique est un contrexemple, la colonisation d'une partie du continent américain ayant fait depuis le $\mathrm{Xv}^{\mathrm{e}}$ siècle de la langue du pays d'origine, le castillan, l'« espagnol », la langue officielle des pays conquis. L'émigration des Espagnols vers les pays d'Amérique latine au $\mathrm{xx}^{\mathrm{e}}$ siècle est la continuation d'un «habitus migratoire ", les émigrants étant cette fois non plus en position de découvreurs ni de conquistadores colonisateurs dominants, mais d'émigrants cherchant des débouchés dans les anciennes colonies qui pratiquent la même langue.

$2 \mathrm{Au}$ début du $\mathrm{XX}^{\mathrm{e}}$ siècle l'émigration vers la France déjà commencée au $\mathrm{XIX}^{\mathrm{e}}$ siècle est accélérée par la première guerre mondiale ; migrations économiques s'il en est, elles fournissent une main-d'œuvre agricole saisonnière au sud de la France. Des réseaux d'émigration/immigration sont constitués et dans les années 1920, les Espagnols sont la $3^{\mathrm{e}}$ nationalité vivant en France. Limitée par les politiques françaises d'immigration en raison de la crise de 1929 et coïncidant avec l'avènement de la II ${ }^{\mathrm{e}}$ République en 1931, la migration change de sens et les Espagnols vont revenir dans leur pays. Cinq ans plus tard, en 1936 la victoire du Front populaire aux élections générales consolide la République mais les problèmes sociaux sont tels et les attentes de changements si fortes pour les classes défavorisées que les droites aux aguets se soulèvent sous l'impulsion du général Franco. La guerre civile éclate en juillet 1936. Dès le début et jusqu'en 1948, un exode inédit se produit. Plus de 500000 réfugiés passent la frontière française. La fermeture de la frontière franco-espagnole à l'initiative du gouvernement français du $1^{\mathrm{er}}$ mars 1946 au 5 février 1948 suscitera non l'arrêt des passages, la frontière n'étant pas totalement étanche, mais la constitution de réseaux d'émigration 
clandestine. Près de 23500 réfugiés clandestins continuent d'entrer en France entre 1946 et 1952, notamment des guérilleros exfiltrés ${ }^{1}$, auxquels se mêlent les «économiquement faibles" refoulés à la frontière à partir de 1948. À partir des années 1960, aux motifs politiques se substituent massivement les impératifs économiques et on dénombre près de 100000 immigrants en 1960 quand des accords bilatéraux sur les migrations sont signés avec la France ${ }^{2}$. Ce mouvement coïncide avec les énormes besoins de main-d'œuvre pour la reconstruction, en France et dans tous les pays d'Europe, qui absorbent l'hémorragie démographique espagnole. Les Espagnols constituent la deuxième population immigrée la plus importante après les Italiens tant en France qu'en Allemagne ${ }^{3}$. En 1956, le pouvoir veut reprendre le contrôle des flux migratoires et crée l'Institut espagnol d'émigration (IEE). L'ouverture des frontières produit l'une des vagues migratoires les plus importantes de la longue histoire des migrations espagnoles, la grande vague des années 1960 . Entre ces deux grandes vagues, 1948 et 1960, les migrations intérieures se sont poursuivies. Moins nombreuses, elles atteignent cependant leur niveau le plus élevé $\mathrm{du} \mathrm{xx}^{\mathrm{e}}$ siècle et constituent un chapitre important de l'histoire des migrations espagnoles et contribuent à la configuration de l'Espagne contemporaine.

3 Si les grands flux migratoires $d u x^{e}$ siècle vers les anciennes colonies n'impliquaient pas un effort linguistique important pour les migrants espagnols, il n'en fut pas de même pour la "grande vague » des années 1960 vers la France, l'Allemagne, la Suisse, ou le Royaume-Uni, dont la langue leur était totalement étrangère. Dans ces pays, des politiques linguistiques accueillantes ont pu déterminer un fort taux d'intégration des immigrés :

Si l'immigration espagnole ressemble à celle en provenance des autres pays d'Europe du Sud, en ce qu'elle concernait essentiellement des travailleurs manuels faiblement qualifiés, parfois analphabètes, elle s'en distingue de façon notable par son ampleur et par la manière dont les Espagnols, une fois installés dans leur pays d'accueil, ont tenté de s'adapter à leur nouveau milieu, tant sur le plan linguistique que culturel et professionnel. Les Espagnols [...] se sont fondus dans la population d'accueil au point de ne plus apparaître dans les statistiques trente ans plus tard. ${ }^{4}$

4 La migration met les individus en situation de devoir apprendre une langue du jour au lendemain, la plupart du temps sans préparation, et par eux, elle met les langues en contact. La nature même des langues qui exercent leur influence les unes sur les autres fait que des parlers hybrides s'inventent. Ils constituent les traces de la contrainte linguistique dans la migration.

5 Montrer que ces traces, tant dans les langues initiales que les langues acquises, et comprendre comment les migrants espagnols, exilés politiques ou migrants économiques se sont confrontés à cette réalité, est l'objet de cet article qui s'inspire des travaux de la sociolinguistique sur le contact des langues dans la migration. Avec ses quatre langues historiques devenues co-officielles dans la constitution de 1978, l'Espagne et ses migrations séculaires, est un véritable laboratoire sociolinguistique.

6 Dans une première partie nous retracerons quelques aspects du développement de la sociolinguistique, notamment en Espagne où les études sur le bilinguisme, ses causes, origines et ses conséquences, notamment dans les migrations se sont développées. Dans une deuxième partie, nous présenterons plusieurs exemples de mise en contact des langues d'Espagne à travers des locuteurs ayant vécu l'exil ou la migration, contact du galicien et du catalan, de l'espagnol avec d'autres langues comme le français ou 
l'allemand. Enfin, la troisième partie présentera des exemples de traces des migrations galiciennes dans le castillan ou le catalan.

\title{
Théorie linguistique, sociolinguistique et langues en contact
}

\section{La langue et le langage}

\section{De la compétence langagière à la langue}

7 La compétence langagière caractérise l'espèce humaine et le langage est le premier outil relationnel entre les individus. En tant que "communicant émotionnel », l'être humain réalise la "pulsion d'interliaison » postulée comme «contraignant les individus en présence à une participation énergétique réciproque. $»^{5}$ "Si [...] l'enfant n'est pas seulement guidé par le plaisir, mais par une nécessité participative avec l'environnement, il deviendra le siège d'un énorme trafic interne-externe. $»^{6}$ Par ce «trafic » l'individu réalise ses premiers contacts avec l'autre, les autres, le monde, pour s'y inscrire en tant qu'autre. Ces échanges sont immédiatement soumis à la structure d'un langage. En 1916, Saussure fut le premier à définir la langue en la différenciant essentiellement du langage, ouvrant ainsi de larges perspectives :

\begin{abstract}
Mais qu'est-ce que la langue ? [...] à la fois un produit social de la faculté du langage et un ensemble de conventions nécessaires, adoptées par le corps social pour permettre l'exercice de cette faculté chez les individus. [...] le langage est multiforme et hétéroclite [...]. La langue, au contraire est un tout en soi et un principe de classification [...] une chose acquise et conventionnelle. [...] et la nature du signe dont on est convenu est indifférente. ${ }^{?}$
\end{abstract}

8 L'enfant rencontre le corps social par le langage et la langue est la ressource à acquérir. Comme telle, elle entre dans un processus de production et de reproduction, de production d'idées, de discours, d'idéologies et de reproduction des pouvoirs ${ }^{8}$. Cette vision du travail des langues amène à penser la question des relations entre les langues en termes de domination, tout particulièrement dans la migration, où les langues se retrouvent en contact dans une hiérarchie liée aux motifs de la migration.

\section{Le contact des langues}

9 La sociolinguistique moderne a montré dans les années 1980 du $x^{e}$ siècle que l'évolution des langues tient autant à leur hypothétique nature qu'à leur dimension sociale : elles vivent et meurent au sein de groupes sociaux, en fonction des nécessités d'interactions individuelles ou collectives.

\section{Le mélange est la nature des langues}

10 La notion de mélange apparaît comme la nature même de l'activité linguistique. Le mélange se constitue à partir d'un substrat compris comme l'influence ou l'«interférence entre systèmes impliquant la modification des catégories (grammaticales ou sémantiques) ». Il se différencie de l'emprunt ${ }^{9}$ lexical qui introduit un élément nouveau dans le système existant « sans conséquences pour le système de la langue qui les incorpore $»^{10}$. Le substrat n'a pas d'existence biologique ; il est la trace de processus longs entre des membres de ce qui devient une communauté linguistique - 
par exemple le latin est le substrat des langues romanes -, et un phénomène analysable du point de vue social. Les langues se superposent, s'influencent mutuellement, ne disparaissent qu'en laissant des traces sur celle(s) qui les domine(nt). Si elles sont des organismes « vivants ", elles meurent aussi.

Ouvrant la perspective sociale à l'étude de l'évolution des langues, la sociolinguistique s'oppose à cette théorie organiciste qui donne lieu au structuralisme. Le changement n'est plus «une "altération" ni une "détérioration", [...] mais reconstruction, rénovation du système, en assurant la continuité et le fonctionnement. »"11

Une langue est finalement une structure et des mouvements liés à des influences internes (usure, loi du moindre effort ou principe d'économie ${ }^{12}$ ), ou externes (contact avec d'autres milieux linguistiques). "La langue est un système en mouvement ${ }^{13}$, elle est "un processus social et même un fait social». Le concept d'économie de la langue désigne ce "système combinatoire » universel qui permet de construire «à partir d'un nombre limité d'éléments (morphèmes et phonèmes) un nombre illimité de mots et de phrases, dans toutes les langues. ${ }^{14}$

13 Les langues ont d'abord été pensées comme systèmes homogènes - la perspective synchronique privilégiée par Saussure -, leurs changements venant d'un substrat, notion elle-même relativisée par la reconnaissance des changements comme constitutifs des langues. Les concepts d'interférence ou de variation, phénomènes capables de diversifier et de modifier les langues dans leurs catégories parlées ou leurs structures profondes au contact d'autres structures linguistiques, vont permettre de nouveaux développements. D'autres concepts d'« hétérogénéité », d'« hybridité », de variété ou de "parenté multiple ${ }^{15}$ entrent dans le lexique de la linguistique devenue sociale.

\section{Des langues à la langue, le produit des contacts}

\section{Monolinguisme, bilinguisme, multilinguisme}

La situation de monolinguisme d'abord considérée comme la situation « naturelle » est en fait l'exception :

[...] le bilinguisme est un phénomène qui affecte la plupart des populations de la terre. [...] La vision actuelle des linguistes qui considèrent le monolinguisme comme la norme et le bilinguisme ou le plurilinguisme comme le phénomène exceptionnel, ne correspond pas à la réalité [...][où] pour satisfaire les nécessités de communication, la majorité des locuteurs de la terre connaît et fait usage de plus d'une langue. ${ }^{16}$

L'unilinguisme est analysé comme une idéologie des sociolinguistes basée sur des représentations erronées: hiérarchie des langues (le "génie» des langues), représentation politico-administrative et «représentation élitiste de LA langue ainsi posée comme idéalement immuable, inaltérable, indépendante pour ainsi dire de la communauté d'usagers ${ }^{17}$, qui contredisent une réalité bien plus complexe : plus de six mille langues sont " parlées aujourd'hui sur la planète $»^{18}$, l'Union européenne possède « vingt-quatre langues officielles et de travail »" ${ }^{19}$, mais il y a aussi « près de soixante-dix "langues régionales ou minoritaires" à l'intérieur de l'UE » et même 75 langues de France $^{20}$; par ailleurs l'existence d'États multilingues comme la Suisse, modèle dit « harmonieux ", ou l'Espagne des autonomies, modèle plus "conflictuel », toutes ces 
réalités suggèrent l'importance des politiques linguistiques dans le développement et l'évolution des langues et de leurs contacts.

\section{Influences et résistances} plupart du temps lexicaux ${ }^{27}$. Mais au-delà du lexique, les points faibles structurels favoriseraient les interférences, de même que la stabilité serait un facteur de résistances. ${ }^{28}$ Mais on n'observe aucune systématicité des interférences, leur possibilité étant rapportée à une multiplicité de facteurs parmi lesquels, en dehors de qualités propres, les conditions de leur production, la situation des locuteurs, psychologique, sociale et leur implication dans les phénomènes linguistiques :

L'impact total des langues les unes sur les autres peut difficilement être pris en compte dans les données strictement linguistiques. Dans pratiquement toutes les formes d'interférence, il y a un jeu de facteurs extérieurs aux structures des langues qui favorisent ou inhibent le développement de l'interférence. Les facteurs externes comprennent les traits individuels des locuteurs bilingues, les circonstances dans la situation d'élocution et le contexte socioculturel du contact de langue, parmi lesquels la valeur sociale, le purisme, et des considérations similaires sont opérantes. L'élément temporal ou la durée du contact peut aussi être en jeu. ${ }^{29}$ un processus de bilinguisation, les mêmes processus sont en jeu. 


\section{Les formes du bilinguisme} conflictuel, la situation de diglossie. Pour le premier, les deux langues jouissent d'un statut partagé voire identique; il est en vigueur dans un État plurilingue comme la Suisse qui a trois langues officielles. Le second, le modèle diglossique, a pour modèle l'Espagne aux quatre langues historiques co-officielles selon la Constitution de 1978, la Catalogne apparaissant comme un " laboratoire en matière de politique linguistique. $»^{32}$ Le conflit linguistique ou diglossie de la Catalogne a révélé des enjeux politiques, culturels et sociétaux; il a contribué à "forger des instruments d'analyse [de la] sociolinguistique périphérique » ou "groupe des linguistes "natifs" » qui ont amené les débats sur les situations de minoration linguistique ${ }^{33}$. La sociolinguistique catalane a ainsi fait évoluer le concept de diglossie le faisant passer d'une "représentation fonctionnaliste statique » à " une représentation beaucoup plus dynamique » :

d'une polarité sociolinguistique neutre on va passer à une polarité problématique entre une langue dominante et une langue dominée. [...] [Le] dilemme : ou bien la langue imposée va se substituer lentement mais sûrement à la langue dominée ou bien les usagers de celle-ci vont œuvrer à sa normalisation (c'est-à-dire à une utilisation normée dans tous les domaines de la communication) en combattant les tendances à l'assimilation. ${ }^{34}$ (nous soulignons)

Ainsi, la dimension de l'implication sociale des locuteurs et des enjeux politiques des questions linguistiques devient patente dans le contexte de diglossie catalan, dont l'étude s'élargit à tous les contextes plurilinguistiques.

\section{Les bilinguismes en Espagne}

Plus d'un tiers de la population espagnole est bilingue, ce qui constitue le fait linguistique majoritaire, notamment $\mathrm{du}$ fait de l'arrivée constante de populations originaires d'autres pays ${ }^{35}$. De plus, les situations de bilinguisme et « les interrelations entre les groupes linguistiques en contact " tendent à multiplier les contacts entre langues différentes et "entraînent l'acquisition par de nombreux locuteurs de répertoires et de pratiques plurilingues $»^{36}$.

La situation de diglossie du catalan dominé par le castillan est intéressante dans notre perspective de comparaison entre langues en contact dans la migration, avec un modèle ici en quelque sorte inversé, le catalan se trouvant en position de langue dominante tandis que le galicien est la langue dominée, le castillan jouant le rôle crucial de langue véhiculaire.

\section{Contact du galicien et du catalan, une « transculturation » à bas bruit ?}

En 2015, à partir du concept de "transculturation », nous avons essayé de repérer un phénomène potentiellement à l'œuvre entre la Galice et la Catalogne ${ }^{37}$. Cette émigration, qui n'est pas la plus importante en nombre de personnes déplacées, est néanmoins l'un des flux les plus constants à l'intérieur de la péninsule. Deux langues, deux cultures, deux sociétés y sont impliquées. Ce phénomène est de faible intensité 
mais l'interrogation sur les traces du passage d'éléments linguistiques, de l'une à l'autre langue nous a amenée, sans les outils de la sociolinguistique, à la difficulté de montrer si le galicien, en situation minorée, subissait des influences du catalan, dominant, ou si les deux langues exerçaient des influences mutuelles, ce que nous postulions.

\section{Langues en contact dans les migrations}

La sociolinguistique a mis en évidence l'influence des migrations, qu'elles soient « internes » ou « externes » dans l'évolution des langues :

Les migrations internes (au sein d'un même territoire national : par exemple lors d'un exode rural) ou externes (entre deux pays) sont des situations où ne manquent pas d'apparaître des phénomènes sociolinguistiques orignaux liés aux contacts entre deux ou plusieurs langues (la ou les langues des migrants, la ou les langues du pays d'accueil) dans divers contextes, au sein de communications soit exolingues (entre membres des deux groupes en présence) soit endolingues (entre les membres du groupe des migrants). Ces conditions spécifiques du contact des langues dans la migration produisent en effet chez les migrants (enfants comme adultes) des usages sociolinguistiques à mesure de la modification de leur répertoire linguistique. ${ }^{38}$

Quand elles émigrent, les personnes d'une communauté linguistique sont en contact avec d'autres personnes ne parlant pas la même langue. Il s'agit la plupart du temps d'une plongée dans l'inconnu. Les réseaux de migration sont une modalité sécurisante : quand on part dans l'inconnu, on préfère le moins inconnu. Ce n'est pas le cas pour les migrants pionniers et pionnières qui ouvrent des voies, encore moins pour les réfugiés politiques partant sous la menace de dangers imminents. Les migrants et les exilés se retrouvent en situation de devoir apprendre une nouvelle langue, en subissent la hiérarchie - la leur étant la plupart du temps dominée - et cet apprentissage conditionne l'évolution de leur situation et leur avenir. Elle est le vecteur de l'intégration dans la société d'accueil et s'impose selon les lois du pays.

Considérer l'aspect linguistique de la migration, c'est faire droit à la difficulté que constitue en soi l'apprentissage d'une langue en situation de migration.

\section{Apprendre une langue...}

31 Steiner considérait qu'apprendre une langue, c'est "entrer en possession d'une connaissance active de la réalité [...] La langue est ce "quelque chose" de fondamental dont la perte entraîne celle de l'identité vivante. ${ }^{39}$ L'objet de cet article étant d'observer ce "quelque chose » dans la migration comme circonstance qui impose le contact des langues, on peut se demander comment les langues elles-mêmes "gèrent " ce contact labile ${ }^{40}$, comment les individus traversent ce qui constitue une épreuve souvent relativisée dans leurs témoignages. La capacité à se débrouiller ou la nécessité de la survie sont des enjeux vitaux pour l'émigrant, souvent avant-même de quitter la terre natale. La quête d'une identité individuelle et sociale nouvelle, fantasmée puis intégrée ou restant toujours entre deux mondes, hybride, se joue dans l'apprentissage et rejoue des déclinaisons de "la double absence ${ }^{41}$, toujours au prix d'une perte, et devient centrale dans leur « apprentissage actif de la réalité ». 
Les « interlangues » des migrants

\section{Hybrides, pidgins} sociales en général influent sur l'évolution des langues. Mais dans quel sens se fait cette influence? Si c'est dans un seul sens, alors est-ce dans celui qu'impose la langue acquise, dominante? Les problèmes spécifiques qui se posent du fait même de la migration, la nécessité de communication, la réalité du contact, produisent des parlers hybrides en situation de domination. L'«interlangue » créée au contact de la langue à acquérir - basée sur les interférences dans le discours, les variations introduites par les locuteurs - est considérée comme un "outil linguistique instable et éphémère », un « outil pragmatique qui se soutient de la nécessité du migrant de communiquer dans une langue qu'il ignore à des degrés divers ", qu'il maîtrise mieux avec le temps et en fonction de son investissement et qui se propage en fonction d'un marché linguistique ${ }^{42}$, c'est-à-dire des nécessités de la communauté linguistique sur un territoire donné, en un temps donné. La situation de migration produit chez les locuteurs "des usages sociolinguistiques spécifiques [...] à la mesure de la modification de leur répertoire linguistique $»^{43}$ car ils sont 《le lieu exact où se produit le contact. $»^{44}$

Notre questionnement visant à comprendre comment l'expérience de la migration transforme la «locution" en même temps que le locuteur, comment de nouvelles langues, transitoires autant que le sont les situations des migrants, s'inventent par interpénétration, influence mutuelle ou de domination, aboutissant parfois à la disparition de telles langues réputées faibles ou à l'expansion de celles qui seraient plus fortes. Après avoir posé un cadre théorique très général sur la problématique des langues en contact, nous allons présenter des exemples choisis de modifications pour des personnes impliquées dans des processus migratoires et d'exil en Espagne.

\section{Langues en contact dans l'exil politique et les migrations économiques sous le franquisme}

Nous présentons des témoignages de migrantes ${ }^{45}$, d'exilé-e-s politiques, pris dans la littérature, le théâtre, ou la réalité des résistances au franquisme. Ils partagent, outre celle du déracinement, l'expérience d'un apprentissage linguistique et nous permettent d'approcher une part des réalités et des difficultés qu'ils et elles ont rencontrées dans les contextes particuliers de l'exil politique ou des migrations économiques entre 1940 et 1970 .

En effet, le changement violent de régime entre 1936 et 1939, de la deuxième République à la dictature, a marqué le point d'arrêt des politiques linguistiques favorables aux langues historiques de l'Espagne. Les Statuts votés par les institutions de la République (le 16 juillet 1936 en Galice, jamais appliqué ; dès 1932, pour le catalan et le 6 octobre 1936 pour le basque), sont abrogés dès 1939, combattus et dégradés comme toutes les libertés individuelles. 


\section{Exilées républicaines}

\section{Le " fragnol », interlangue des réfugiés politiques et des migrants espagnols en France}

Un roman ${ }^{46}$ "con tintes autobiográficos" ${ }^{47}$ (" aux traits autobiographiques») de Lydie Salvayre permet d'observer comment une exilée politique en 1936 a fabriqué une interlangue dans les nouvelles circonstances de sa vie. Ce "frañol, immédiatement compréhensible pour quiconque a fréquenté des exilés en France, constitue un exemple paradigmatique des réalités linguistiques des migrants.

L'acronyme fragnol, («français» plus »espagnol»), utilisé depuis les premières immigrations d'Espagnols en France au XIX siècle, est "généralement vu comme une forme d'expression minoritaire et incorrecte par ceux qui [...] croient dans la pureté des langues ${ }^{48}$.

Dans le roman, le fragnol donne littéralement corps à la situation et au personnage :

[...] très fortement inspiré de ma mère et qui est arrivée en France en 1939 et qui ignorait absolument tout de la France, du français, de sa géographie, de son histoire, de ses mœurs et qui a donc fabriqué une langue mezclée, comme elle aurait dit, une langue mixte, une langue qui a été le résultat du carambolage entre son espagnol de naissance et la langue qu'elle découvrait, [...] que nous appelions, ma sœur et moi, le fragnol. ${ }^{49}$

39 Sorte de pidgin ${ }^{50}$, basé sur l'alternance de mots et de structures des deux langues ou code-switching dans les études sur le bilinguisme. Un relevé de formes exemplaires permet de comprendre certaines stratégies d'énonciation du personnage que nous allons présenter.

Montserrat Monclus Arjona, mère de la narratrice qui tente de raviver, dans son grand âge, la mémoire des années de la guerre d'Espagne et de son exil en 1936, se souvient de son arrivée comme réfugiée sur le territoire d'une France peu hospitalière aux Républicains, en particulier sous le régime de Vichy. Le parler de sa mère devenant immédiatement un personnage à part entière : « elle évoque pour moi sa jeunesse dans cette langue mixte et transpyrénéenne qui est devenue la sienne depuis que le hasard l'a jetée, il y a plus de soixante-dix ans, dans un village du Sud-Ouest français $»^{51}$. Cette interlangue est bricolée à partir de connaissances très limitées du français et dans la contrainte d'acquérir la langue du pays en procédant par contaminations successives et multiples d'espagnol et de français. Il va lui permettre de s'exprimer dans un effort constant et durable de communication. L'exil est d'abord vécu comme provisoire, mais Lydie Salvayre se souvient dans un entretien que, dans la communauté qu'elle fréquentait enfant à Auterive près de Toulouse, "on organisait de grands repas collectifs, on racontait des histoires salaces et personne n'achetait de meubles parce que tout le monde croyait qu'il finirait par revenir vers la patrie abandonnée. $»^{52} \mathrm{La}$ plupart du temps, comme pour elle, l'exil n'a pas été provisoire.

41 Nous allons observer quelques-uns des "bricolages " et procédés de cette langue hybride. Comme le pidgin, elle est constituée d'une «alternance de plus d'une langue dans un même langage $»^{53}$. Les éléments de l'autre langue apparaissent soit à l'intérieur d'une même phrase soit en phrases entières qui alternent. Dans l'ouvrage, on relève pour chacun des modes respectivement cent soixante-dix-neuf et vingt-neuf occurrences ${ }^{54}$. À plus de $90 \%$, le mélange des mots ou expressions se fait à l'intérieur 
des phrases, sur la structure du français. La préoccupation de Montse est bien d'être comprise, ce qui détermine les stratégies privilégiées.

Voici quelques exemples : «sans le moindre remordiment", (p.78) est une création sur la base de l'espagnol remordimiento, le «remords"; "José s'en va sans arrepentiment (dit ma mère)»(p.79), formé à partir de arrepentimiento, dit les « regrets »; « les vieux paysans s'équivoquent » (p. 79), pour « se tromper » à partir du verbe espagnol equivocarse, réalise un parfait parallélisme avec le français « équivoque " pour lequel il n'existe pas de verbe; enfin, "Ils éprouvèrent l'un pour l'autre de l' amistad (ma mère me dit qu'en espagnol le mot a davantage de panache, soit.) » (p. 226) : cette dernière occurrence est assortie d'une parenthèse explicite, intervention de la narratrice et acquiescement de l'auteure, qui signale la supériorité (ressentie) de l'espagnol dans le registre émotionnel et précise que, pour le personnage comme pour l'écrivaine, quelque chose de leur identité s'exprime.

Le texte compte vingt-neuf occurrences du procédé d'alternance de phrases françaises et espagnoles; en voici trois exemples pour illustrer notre propos : "Un angel del cielo " (p. 55), "Cuidado con el pelirrojo !" (p. 146) (présente de plus une alternance interne de ponctuation avec l'absence du point d'exclamation initial), et "Qué suerte, qué felicidad!" (p. 168).

D'autres procédés parsèment le texte comme des calques, des déviances sans influence ou par contamination de l'espagnol. Le calque, qui présente « une orthographe et une morphologie correctes $\|^{55}$ est une traduction littérale. Il y a 16 calques dans le roman on pourrait dire : seulement. Le titre du roman, Pas pleurer, est un calque parfait de l'expression espagnole "no llorar", - titre de la version espagnole du roman ${ }^{56}$. En français standard, on aurait dit «Ne pas pleurer» («je m'intime l'ordre de ne pas pleurer, je ne dois /veux pas pleurer »), le choix du calque nous paraît condenser la problématique linguistique du roman et, à travers elle, celle de la résistance qu'oppose le personnage, dans la situation supposée provisoire de l'exil, à la violence que celui-ci lui a faite. Ce choix affirme la force de caractère qu'il faut pour s'adapter sans montrer sa souffrance, comme une politesse de l'hôte envers l'hôte.

Enfin, « [...] à toute vélocité vers mon grenier » (p. 14) traduit une autre dimension du pidgin, l'usage de mots désuets par le fait de racines communes.

Deux exemples de déviances, ou formes fautives, d'abord sans influence de l'espagnol: "la guerre ma chérie est tombée à pic nommé" (p.15) où se mélangent les deux expressions, «tomber à pic » et "à point nommé », ou « je le dis sans l'ombrage d'un doute» (p. 79), mélange de "sans l'ombre d'un doute» et, peut-être, "sans ambages » ou "sans en prendre ombrage ", dénotent aussi le risque que prend la locutrice dans un évident souci de correction, sans l'appui de la langue maîtrisée, contrairement au calque directement importé de l'espagnol. Déviance enfin, sous l'influence de l'espagnol dans des formes linguistiques complexes, l'expression « As-tu comprendi qui étaient les nationaux? » (p. 94) : celle-ci combine les structures verbales comprendiste, et "compris», participe passé irrégulier méconnu du personnage. De même, «nos yeux se saludèrent et l'amour se leva» (p.147), se saludaron et " saluèrent ». Pour finir, une trouvaille, dans les derniers mots du roman, situe Montse dans toute l'étendue de son instabilité linguistique, de son « inquiétude $»^{57}:$ «Si tu nous servais une anisette, ma chérie. Ça nous renforcerait la morale. On dit le ou la? » Et sa fille de répondre: "On dit le. Le moral. $»^{58}$ rendue avec assurance, au rôle de correctrice, celui de sa génération. 

communautés linguistiques diverses constituant un véritable champ de la recherche sociolinguistique. Le parler "Melandjao" des immigrés de langue espagnole en Roussillon ${ }^{61}$ est un autre exemple d'«outil linguistique instable et éphémère " créé par des Espagnols. Le "melandjao» est un produit du contact interlinguistique, le terme qui intrigue " émanant des propres locuteurs de ce mode d'expression: "Nosotro' hablamo' melandjao". Les immigrés de langue espagnole de la première génération, qui résident [dans le Roussillon] depuis parfois plus de trente ans", pratiquaient cet interlecte «assez récemment supplanté dans l'intégralité de ses usages $»^{62}$ par le français. Le terme melandjao « unit opportunément le fond à la forme, (signifié et signifiant) ». Il est l'interférence même «entre ces codes qui se brouillent, se parasitent l'un l'autre. [...] Melandjao est donc la projection d'une forme grammaticale castillane [...] sur un contexte francophone. ${ }^{63}$ linguistiques qui les produisent. Le Melandjao s'est perdu avec le tarissement de l'immigration espagnole saisonnière, retournée au pays ou assimilée, qui a laissé place à d'autres immigrés, possédant probablement leurs parlers hybrides de migrants.

51 Ces premières personnes soumises au contact des langues avaient reçu une formation scolaire limitée, voire n'en avaient pas eu du tout. Le milieu social influe directement sur la qualité de leur apprentissage. Une meilleure condition sociale évite-t-elle l' interlangue?

\section{Maria Casarès, réfugiée politique et " Résidente privilégiée "}

Lorsque la guerre civile espagnole éclate, Maria Casarès ${ }^{64}$ et sa mère sont envoyées à Paris par le père, Santiago Casares Quiroga, ministre du Gouvernement de la République démissionnaire. La famille Casarès ne faisait pas partie des «économiquement faibles" et Maria doit impérativement pouvoir poursuivre ses études, ce que la guerre et le danger n'auraient pas permis. Également issu pour nous 
du champ littéraire ${ }^{65}$, l'exemple de l'apprentissage du français par la célèbre comédienne de la scène française des années 1940 à 1980, rappelle qu'elle était une exilée politique espagnole, originaire de Galice. Maria Casarès rapporte dans son autobiographie ${ }^{66}$, l'expérience d'un exil brutal, mais d'une expérience linguistique et vitale exceptionnelles. A Paris, elles ne connaissent personne et ni l'une ni l'autre ne parle le français. Maria a treize ans. Cependant, « par l'entremise de Jean Cassou » elle a très rapidement une place dans une «classe Babel » de jeunes étrangères «[...] ballotant selon les continuelles arrivées et les continuels départs entre douze et quinze [élèves] au maximum $»^{67}$. Des cours singuliers ont lieu dans un pavillon :

Au fond des jardins du lycée Victor-Duruy, avoisinant le musée Rodin, il y avait un petit pavillon consacré uniquement aux étudiantes étrangères, et où la très gentille madame Wurmser déclamait à tue-tête, avec gestes à l'appui, des textes de Corneille ou de Hugo. ${ }^{68}$

Contrairement à Montserrat Monclus Arjona, Maria apprend le français par l'injonction familiale de poursuivre ses études, et « il s'agissait d'apprendre à connaître une langue, ses écrivains, ses poètes $»^{69}$, par la lecture de grands textes des grands auteurs de la littérature française, en les déclamant! Ce «plaisir des textes» restera la caractéristique de la comédienne. Mais cela n'empêchait ni le «baragouinage » ni la difficulté éprouvée :

Parfois cependant, avec beaucoup d'efforts, nous baragouinions entre nous ou avec Mme Wurmser un charabia qui se voulait du français - il le fallait bien ! on était là pour ça! mais un français assaisonné de déformations et d'accents si abracadabrants, que l'on se demandait comment, dans un tel bouillon, on pouvait arriver à un résultat. On y parvenait pourtant. (Nous soulignons) ${ }^{70}$.

Le «français assaisonné de déformations et d'accents abracadabrants", c'est «la langue hybride et transpyrénéenne » ou le «melandjao » d'autres migrants ou réfugiés d'une origine sociale plus modeste. La jeune Maria en est consciente et elle est mue par la forte nécessité qu'elle éprouve de bien parler. Son témoignage est un exemple d'apprentissage dans le cadre scolaire, mais en même temps, elle découvre sa vocation théâtrale qui allait l'emporter. Dans un entretien radiophonique, elle évoque sa passion pour la vie et la formidable nécessité qui la pousse :

En fait je n'étais [...] consciente que d'une chose pendant ces 5 ans, d'abord apprendre le français, ensuite suivre les cours de la $3^{\mathrm{e}}, 2^{\mathrm{e}}$, et $1^{\mathrm{e}}$ de baccalauréat et de philo ensuite, et d'autre part d'aller dans tous les cours de diction et d'art dramatique [...] pour travailler et apprendre le théâtre. ${ }^{71}$

En cinq ans, elle passe d'exilée ne connaissant pas le français à l'obtention de deux prix du conservatoire et à des débuts fulgurants au théâtre. Modestement elle analyse : « Je crois qu'il y a eu là une espèce de violence de volonté... qui s'est éveillée peut-être du fait du départ de l'Espagne... ».

La pédagogie consistant à laisser la nouvelle langue prendre corps, physiquement, pour les nouvelles arrivées à l'écart des autres élèves, puis de leur faire rejoindre le cursus normal quand l'acquisition est suffisante, lui semble exceptionnelle pour un bon apprentissage. En très jeune exilée elle entre dans la langue française avec un bonheur qu'elle ne se lassera jamais d'évoquer et parle du sentiment de liberté dont elle fait l'expérience dans un environnement qu'elle sait privilégié :

Le pavillon d'étrangères du lycée Victor-Duruy était encore un de ces lieux privilégiés [...] où le respect du prochain, et de ce qu'il apporte d'inconnu, mesurait la liberté de chacun jusqu'à rendre la liberté de tous, sinon totale, du moins bonne à vivre. $^{72}$ 

produire, l'interdiction pour des milliers d'exilés de franchir la frontière: "Cette liberté ne s'arrêtait pas à la porte du pavillon; elle passait les frontières et [...] nous n'avions qu'à brandir les cartes d'“étrangère" pour passer la frontière sans problème. »

L'apprentissage de la langue dans le cadre scolaire semble procurer à l'exilée un quasibilinguisme et un bonheur évident. Mais pour clore le chapitre consacré à l'exil, nous allons évoquer une autre situation dans l'exil de la dernière heure, celle de résistants au franquisme restés en Espagne aussi longtemps que possible.

\section{Francisco Martínez-López dit « El Quico »}

Francisco Martínez-López est un guérillero antifranquiste. Il a mené et tenté d'organiser une résistance au régime de Franco dans la région du Bierzo Galice. Il rapporte dans le huitième et dernier chapitre, "L'exil », de son témoignage naturaliste et poignant, les événements qui ont émaillé sa sortie du territoire espagnol en compagnie de trois autres guérilleros, «El Atravesado», Manolo et «El Jalisco» en septembre 195173. Ils craignent d'être "déportés en Corse comme l'ont été à cette époque de nombreux militants du PCE déjà installés en France». Au commissariat de Bayonne, ils sont soumis à un interrogatoire et le commissaire confirme que « les bandits communistes sont des délinquants de droit commun et n'ont aucun droit à l'asile politique ». La question linguistique n'est pas évoquée. Ils ont bon espoir d'obtenir gain de cause, un réseau d'exfiltration des guérilleros étant déjà organisé en France, mais leur situation est très précaire :

Nous [...] sommes très loin de savoir évaluer la situation politique en France et d'imaginer que nous sommes à deux doigts d'être livrés, menottes aux poings, à la police franquiste par la police française, pour être garrotés dans une prison du León, comme ce fut le cas de notre camarade Marcelino de la Parra en 1948.

Militants internationalistes actifs, ils sont peu préoccupés par les questions de communication. Lors d'une interview réalisée en 2019 chez lui, "Quico » confirme : «de toutes façons [sic], avec les mains on se faisait toujours comprendre, c'est une langue internationale!». Quand ils se retrouvent en situation de contact, les moments de communication sont, à deux exceptions près, collectifs : «Tandis qu'il explique en français au commissaire qui sont ces quatre personnages désarmés et confiants, Amadeo dépose nos armes, une à une, sur son bureau. » Le commissaire décidera quand même de les envoyer à Marseille où s'embarque à ce moment-là la légion: "Ni les questions que nous posons, ni les explications données par Amadeo n'y peuvent rien : nous entreprenons ce voyage forcé sans rien savoir de notre destin. $\aleph^{74}$ Dans les échanges, toujours dans l'urgence, il semble que tout le monde comprenne, qu'il y ait toujours un guérillero qui maîtrise le français, ou un policier l'espagnol :

Dès mon arrivée, le commandant de la place me présente des imprimés déjà remplis à mon attention : je n'ai plus qu'à les signer pour être enrôlé dans la légion. [...] Je fixe le commandant et lui rétorque : "vous parlez sérieusement? Vous savez bien que je suis ici de force et que j'attends l'annonce de ma libération par le ministre de l'Intérieur." Sans plus de commentaires, je m'obstine à refuser. Le commandant devient rouge de colère et crie dans un espagnol parfait: "Je te jure que Franco te coupera les couilles".

61

Quand la libération est enfin en vue, il se retrouve seul pour récupérer un laisserpasser :

L'Âge d'or, 12 | 2019 
Je vais le demander dans le bureau du commandant, en prétextant - dans un baragouin moitié français, moitié espagnol - que je dois laisser une valise dans un bar. [...] Le commandant appelle alors un légionnaire et lui ordonne de m'accompagner.

[...] Quand nous parvenons au bar, le légionnaire, qui ignore tout de ma situation, me sert d'interprète : il demande si je peux laisser ma valise pendant deux jours, pour

la durée d'un aller-retour à Marseille. (Nous soulignons)

C'est la seule allusion aux difficultés liées à la méconnaissance du français. Et cela ne l'arrête en rien si près du but. Il est mu par une vitalité hors norme et une prudence innée qui lui permettaient, dans des conditions d'un danger politique extrême, d'organiser la résistance dans les villages du Bierzo-Galice. Réfugié en France jusqu'à la loi d'amnistie (1977), resté fidèle à ses engagements politiques mais aussi au pluralisme caractéristique de la résistance dans le Bierzo Galicia, il a notamment animé le siège du PCE en France. La langue dont il a dû faire usage pendant toutes ces années est sûrement restée une langue mixte. Aujourd'hui, il «baragouine » toujours un français approximatif. Il a entamé sa seconde guérilla " une guérilla pour la mémoire »75 à son retour en Espagne, menant des actions pour revendiquer, au-delà du «droit à une digne sépulture " pour les guérilleros et pour leurs familles qui souvent la réclament, la reconnaissance d'un statut de guérilleros, de résistants antifranquistes, contre le double oubli qui les mène des fosses communes où ils sont anonymes à la tombe et à l'oubli pour l'éternité. Mais ceci est une autre histoire.

63 Pendant ce temps, de guerre et d'après-guerre, des mouvements migratoires se poursuivent à l'intérieur du pays. Notre dernière partie sera consacrée à l'évocation de migrants et de migrantes entre les années 1940 et 1970.

\section{Migrantes galiciennes en Catalogne}

64 Concernée directement par cette migration, nous en connaissions bien certains aspects linguistiques de "contamination » entre les deux, voire les trois langues, le castillan étant la langue véhiculair $e^{76}$. Nous avons rappelé la singularité linguistique de l'Espagne, son multilinguisme ou son pluriculturalisme: $31 \%$ de la population, environ 14 millions de personnes, sont bilingues, pratiquant au moins une autre langue que le castillan. Mais c'est bien, dans son rôle de langue véhiculaire, que le castillan est un facilitateur d'intégration pour les migrants de l'intérieur, et l'une des clés de la « réussite » de l'expérience migratoire.

Les émigrantes galiciennes interviewées ne parlaient pas un mot de catalan quand elles arrivaient en Catalogne entre 1940 et 1950. Elles parlaient le galicien. Elles connaissaient le castillan, «bien sûr!», dit l'une d'elles. D'autant plus que l'environnement immédiatement bilingue est rudimentaire, elles n'identifient pas le castillan comme une langue à part. C'est « l'autre langue ", la première ou la deuxième, selon leur niveau social ou leur région. Leur bilinguisme fluidifie le contact et facilite leur adaptation à leur nouveau monde, urbain et ouvrier. Elles sont très jeunes, poussées hors de la Galice par la misère, mais aussi par la condition particulière des femmes et des benjamins dans la transmission traditionnelle de la propriété : se marier ou partir, l'aîné des hommes héritant seul de l'ensemble du patrimoine.

Bien sûr, la langue n'est pas le seul facteur de leur adaptation réussie : la nécessité de reconstruction après la guerre crée de gros besoins en main-d'œuvre peu qualifiée dans une Catalogne industrielle rapidement redevenue dynamique permet leur embauche immédiate comme ouvrières ou souvent comme servantes au service de familles de la 
bourgeoisie urbaine. Elles témoignent de la confrontation avec la langue étrangère, le catalan, dans les ateliers où les groupes d'ouvrières déjà formés, d'abord exclues comme immigrées et comme Galiciennes c'est-à-dire pauvres, paysannes, souvent illettrées. Mais elles disent aussi comment, en quelques mois, le catalan leur devient familier, se débrouillant à l'atelier, chez le boulanger, sur leur lieu de travail, dans les commerces. Elles se sont coulées avec une relative facilité dans la société catalane, alors accueillante à leur force de travail. De bilingues elles deviennent trilingues.

Il faut pourtant rappeler que le régime franquiste a prétendu interdire les langues considérées comme "régionales": le catalan, le basque, le galicien. Il a réussi à en réglementer l'usage dans la sphère publique. Quelle était la situation du galicien?

Avec l'instauration de la II ${ }^{\mathrm{e}}$ République en 1931, [1]e statut d'autonomie a été adopté le 28 juin 1936. Il a reconnu pour la première fois la co-officialité du galicien et du castillan en Galice. Cependant, durant l'été 1936, le galicien s'est trouvé à nouveau menacé. Bien qu'il n'y ait eu aucune interdiction explicite de son usage en public, il existait une nette aversion officielle à son égard. ${ }^{77}$

Pour les habitants des régions historiques de l'Espagne (Catalogne, Pays Basque et Galice), la défense et le développement de leurs langues ont toujours été au cœur de la lutte pour la reconnaissance de leur identité et de leur singularité. Les langues ellemême sont de véritables « lieux » de la résistance ; interdites dans l'usage administratif par le régime, elles n'ont pu l'être ni dans les ateliers, ni dans le service domestique, ni dans les groupes de jeunes immigrées qui se retrouvaient, le dimanche, sur les paseos par affinités de provenance, comme c'était le cas pour les "bonnes $»^{78}$ galiciennes ou andalouses. Mais la dictature a contribué à minorer les langues historiques qu'il a cherché à dévaluer en langues « locales », « régionales ».

\section{Julia, « pionnière » de sa génération}

Vers 1940, Julia, 14 ans, quitte son village. Tous les voisins se sont cotisés pour aider dans son projet d'émigration l'aînée de la famille la plus pauvre du village où personne n'est riche. Elle se souvient peu, à près de 90 ans, en 2014, des difficultés linguistiques à son arrivée en Catalogne: "Je suis arrivée de Fruxil à Barcelone, à Fruxil c'était le galicien, à Barcelone c'était le catalan. Je me suis retrouvée pareil quand je suis arrivée en Catalogne que quand je suis arrivée en France : je ne connaissais pas un mot $! »^{79}$. Elle ajoute : « Pour moi ça n'a pas été un traumatisme du tout. On me répondait en espagnol ou on me disait "va apprendre le catalan !" Parce qu'ils étaient comme ça les Catalans. Mais moi au bout de trois mois je parlais déjà le catalan. ${ }^{80}$ De même, aucun souvenir d'interdiction: "On ne me l'a jamais interdit... Je ne me souviens pas que c'était interdit... $»^{81}$. Quant au castillan, les limites sont floues :

Moi je parlais le castillan parce que je ne savais rien parler d'autre. Heureusement que je parlais le castillan. Je crois qu'en Galice - et en Catalogne ça doit être pareil ils ne voulaient pas d'espagnol mais en Galice... j'avais bien appris l'espagnol ! / Q. : et le galicien, tu le parlais... / En Galice bien sûr ! ${ }^{82}$

Ces impressions rapportées quelque 60 ans plus tard semblent indiquer que la langue n'a pas été une difficulté, comme ne semble pas en avoir représenté la question de l'intégration, vécue comme facile: «Je me suis très bien intégrée. Je ne sais pas pourquoi mais je ne me sentais étrangère nulle part ${ }^{83}$ L'urgence de la situation économique, la possibilité de travailler dès l'arrivée, laissaient vraisemblablement peu de place à la réflexion ou aux doutes. Julia a pris en marche l'ascenseur social, 
changeant de vie et de monde en émigrant. Elle mesurera la relative distance sociale avec ses voisins restés au village quand elle y retournera pour sa retraite.

\section{Luisa, une émigrante qui choisit}

71 Luisa est une femme au tempérament joyeux, plus âgée que ses sœurs ne l'étaient au moment du départ, car rescapée d'une probable méningite. Elle rapporte qu'elle voyait revenir les émigrées, "si belles, si mignonnes, si grassouillettes!", qu'elle émigre finalement en 1954. Très bien intégrée dans la société catalane, elle raconte des souvenirs joyeux de la Galice lointaine de son enfance, mais elle n'a plus jamais envisagé d'y retourner. Sa fille, née en Catalogne, Catalane revendiquée, a très peu de contacts avec la Galice et son petit-fils, plus aucun. Interrogée sur son intégration, elle évoque son apprentissage du catalan, signe de difficultés dans les premiers temps mais pas insurmontables au regard du gain social de l'expérience migratoire :

Quand je suis arrivée j'ai pensé : tu ne comprendras jamais rien de tout ça... Mais [...] j'ai toujours été, dès que je suis arrivée, avec des Catalans. Comme ça petit à petit j'ai attrapé la langue, à ma façon, comme ça venait, parce que ça fait ça, on a honte parfois de parler catalan en le parlant mal. On préfère ne pas parler... Mais moi non! moi je parlais, et encore maintenant, peut-être qu'au milieu je dis un [mot] en castillan, mais “je veux dire” (elle le dit en catalan) ${ }^{84}$ ça, ça ne m'a pas fait de mal..$^{85}$

C'est chez son premier employeur, au contact de la famille et des enfants dont elle a la charge qu'elle apprend :

Comme tu es [...] servante tu es toujours avec eux et là tu attrapes un mot, puis un autre... Moi j'avais trois enfants (elle dit le mot catalan: "nanos", puis corrige en espagnol $\left.{ }^{80}\right)$, l'un était très petit et je le devais d'emmener à l'école et le petit... comme si j'étais de la maison! Et tu apprends, comme ils parlent toujours ! ${ }^{87}$

Elle ne s'est jamais sentie exclue par les Catalans, dont elle rapporte pourtant qu'ils pouvaient être critiques à l'égard des immigrés: "parmi les gens, il y a toujours quelqu'un qui va parler mal des immigrés, mais moi ça m'est jamais arrivé. ${ }^{88} \mathrm{Et}$ pourtant, les immigrés andalous et galiciens sont les "charnegos" pour les Espagnols ${ }^{89}$.

\section{Maria Luz, une émigrante sans désir d'émigrer}

Née en 1944, Maria Luz est la cadette de la fratrie et comme telle, la dernière à émigrer vers 1960 (Julia 1940, Luisa 1954, le frère cadet 1959). Si dans les années d'après-guerre l'Espagne était ruinée et sa population décimée, dans les années 1950-1960, l'Espagne franquiste connaît son premier grand tournant économique. Dès le début des années 1950, ne pouvant que constater l'échec du modèle autarcique, au bord de la faillite financière, le régime change radicalement et édicte le premier Plan de stabilisation économique le 21 juillet 1959. Bénéficiant du plan Marshal en 1950 - de fasciste elle est vue à présent comme anticommuniste - l'Espagne entre à l'ONU en 1955 ; elle est consacrée au sein du bloc de l'Ouest sans faire officiellement partie de l'OTAN. En 1961, les premiers accords bilatéraux de migration sont signés avec la France, la " grande vague » dite économique commence. L'Espagne s'ouvre, la Catalogne et, dans une moindre mesure, la Galice, évoluent. Pour Maria-Luz, les conditions économiques ne sont pas les mêmes, elle ne ressent aucune nécessité comme ses sœurs, l'aînée en particulier, de se lancer dans l'aventure de l'émigration qu'elle vivra comme un traumatisme. Son récit dépeint une enfance heureuse près de sa mère, de ses parents, à 
s'occuper des bêtes et se consacrant aux tâches de la ferme, parfaitement adaptée aux rythmes de la campagne galicienne des années 1960 qui n'étaient plus aussi rigoureux. Elle évoque son enfance galicienne comme un "manque de tout", mais pas sans " petits bonheurs ». En 1960, elle rejoint le reste de la fratrie émigrée à Manresa : “moi je suis venue parce que c'était une obligation. Ma mère n'aurait jamais pensé que ça me ferait tant d'effet.» Traumatisée par sa mobilité contrainte, sa confrontation avec le monde urbain de la Catalogne sera difficile. Rapidement malade elle sera prise en charge par ses sœurs. Luisa trouvera les deux seuls emplois qu'elle ait occupés: "minyona" (la miñona, la bonne), puis ouvrière dans l'une des plus grandes usines textiles de Manresa, spécialisée en rubanerie, où elle fera toute sa carrière professionnelle.

Son apprentissage de la langue ressemble à celui de ses sœurs mais elle se souvient de la stigmatisation: "on nous appelait les "charnegos" nous qui venions d'ailleurs, " charnegos!", ici il n'y avait que des Espagnols, mais voilà, “charnegos"... »" . Certes elle était Espagnole, mais une "charnega"! D'autres souvenirs rappellent le rapport aux langues en vigueur en Espagne sous la dictature, lorsqu'il a fallu établir son document d'identité et qu'elle se retrouve face aux fonctionnaires :

Certains [te disaient] "Parle-moi en chrétien !... si [quelque chose] leur échappait [en galicien], bien sûr, c'est qu'il y a des gens qui peuvent parler le castillan, mais il $\mathrm{y}$ en a d'autres que non, parce les gens qui viennent d'un village, [ils sont] très fermés tu sais, et voilà, ils vont à la police et... C"était des vrais matons tu sais...91

"Cristiano", un terme qui s'applique à la seule langue officielle du franquisme, le castillan. Elle explique comment "pour [eux] le castillan, c'était nouveau aussi" ("para nosotros era nuevo también el castellano"). N'ayant, comme ses frères et sœurs, fréquenté l'école qu'un ou deux jours par semaine elle avait peu pratiqué le castillan en Galice, et sur l'interdiction du catalan, elle affirme: "jclaro que se hablaba!" souvient de son apprentissage à l'usine, que la compréhension était rapide et que les accents dénotaient l'origine des ouvrières: "se notaba en las lenguas que venías de pueblo" ("Ça se remarquait que tu débarquais de ton village »). Son évocation de l'état intermédiaire (interlangue) est modeste et prolongée jusqu'au présent: “a lo mejor empiezo esas barrejas ${ }^{93} . .$. [...] si es una conversación larga también me encallo pero es igual, [yo] hablo y ya está!" ("je peux commencer ces barrejas... [...] si c'est une conversation longue je peux caler mais ça fait rien, moi je parle et c'est tout!»). Ses mélanges sont composés d'un castillan improvisé sur la base d'une connaissance acquise épisodiquement, d'un catalan quotidien appris sur le tas, donnant lieu à des "catalanades", de mélanges parfois d'usages fautifs, le tout appuyé sur une structure galicienne orale toujours présente. Le bilan de son intégration est mitigé, et sur la difficile question du retour, elle reconnaît un peu douloureusement la perte des illusions :

Si je te dis qu'aller en Galice pour deux mois, aller là où je suis née pour un séjour même court, à Fruxil, y rester deux ou trois mois, oui, mais pour y vivre je suis plus adaptée ici. M'en aller, dire maintenant tu vas vivre à Sarria, ça non, je ne le ferais pas. Ce qui est enthousiasmant aujourd'hui, demain ne l'est plus, c'est fini... on perd beaucoup ses illusions...94

\section{Justa, une Galicienne en Suisse}

Justa est une autre figure d'émigrée galicienne. Avec son mari qui n'a que des emplois précaires, en quête d'autonomie économique par rapport à leurs familles, elle quitte la 
Galice avec la grande vague des années 1960. Leur motivation est claire "aforrar" (voir infra) pour pouvoir construire leur maison. Leur fille, âgée de moins d'un an, reste en Galice aux soins des grands-parents puis en pension. Ils ne la reverront que quelques années plus tard et la séparation, leitmotiv de cette vague migratoire, reste un souvenir douloureux : « la petite elle restait, à neuf mois et demi... je donnais encore le sein... Imagine comme j'étais contente de partir là-bas ! J'ai perdu près de dix kilos... je préfère ne pas y penser... c'est du passé... $»^{95}$ Embauchés tous les deux régulièrement dans l'hôtellerie à Zürs, Canton de Lucerne, ils sont d'abord pris en charge par des employés espagnols et sont rapidement mis au courant de ce que l'on attend d'eux. Elle fera les lits et lui des travaux plus rudes de nettoyage ou portier l'après-midi. Les Espagnols sont recherchés pour leur implication au travail par les agences suisses mais aussi allemandes, ou françaises.

Ils ont fait plusieurs séjours de plusieurs années jusqu'à pouvoir construire leur maison ; après la première « tranche » de migration, ils ont acheté le terrain ; plus tard, pendant qu'il est resté construire la maison, elle est encore repartie. Leur fille a grandi sans eux et en garde un souvenir douloureux. Elle n'est allée qu'une seule fois en Suisse, en vacances. Peu de temps après leur retour définitif, une fois la maison terminée, le mari de Justa est tombé malade et il est décédé en quelques mois.

Si elle nourrit des regrets sur la qualité de vie, Justa tire un bilan mitigé de leur émigration : ils ont beaucoup travaillé avec son mari et elle en était heureuse. La grande exploitation aujourd'hui dirigée par sa fille et son gendre à quelques kilomètres de son village de naissance, A Cervela (province de Lugo), où ils ont aussi rénové la maison familiale, est le signe d'une émigration économiquement réussie. Pourtant, l'évocation de la vie familiale reste sensible tout comme la mort prématurée de son mari.

En Suisse, ils étaient logés dans de petits appartements tout équipés, “¡muy modernos!" («très modernes »). Leur motivation première, "aforrar", - ce mot très souvent prononcé par les émigrants interviewés, qui signifie "épargner ", revient comme le leitmotiv de l'émigration galicienne massive des années 1960 à la fin des années 1970 -, leur a permis un enrichissement matériel certain, mais l'expérience de l'émigration, qui devait durer quelques années, est devenue l'affaire d'une vie.

Son expérience linguistique tient en quelques anecdotes: premier contact avec des intermédiaires parlant l'espagnol, "nos lo explicaron todo" («on nous a tout expliqué »), un contact épisodique avec d'autres Espagnols dans une des associations très développées alors dans les pays d'émigration des Espagnols, ont généralisé l'usage du castillan. L'apprentissage facile d'une autre langue de Suisse, l'italien : "L'italien je l'ai appris tout de suite parce que c'est très semblable au galicien. Tu l'entends à peine que tu l'apprends déjà. A moi on me demandait si j'étais italienne parce que je le parlais sans l'avoir étudié" 96 . Mais elle n'a jamais appris l'allemand, langue officielle du canton de Lucerne, où elle est restée près de neuf ans, malgré les cours organisés par les associations ou les employeurs : " je suis allée à des cours mais j'ai laissé tomber parce que je ne pouvais pas comprendre. En plus j'ai dit pourquoi je vais apprendre l'allemand si je ne le parlerai plus jamais... Je n'en avais pas besoin, pour quoi faire? Moi, en Allemagne... je n'allais pas y retourner." ${ }^{97}$ Une autre émigrée d'un village voisin interrogée sur la même question rapporte : 
Les premiers [temps] rien. Quand réellement tu te trouvais dans des situations difficiles, avec les mains. Dans les grands supermarchés où tu trouvais de tout, tu prenais ce dont tu avais besoin, tu n'avais pas besoin de parler. (nous soulignons).

82 La synthèse de l'émigration de ces années, "il n'était pas nécessaire de parler »; la question de l'intégration ne se pose que marginalement pour une émigration de travail avec peu ou pas de contacts avec les autochtones, peu de communication sauf professionnelle, peu de distractions, seulement une force de travail régulée. Malgré ces drôles de conditions, Justa reconnaît qu'elle serait restée en Suisse si...

Les Galiciens, on est très nostalgiques de notre village, mais moi je serais restée làbas et j'aurais emmené ma fille là-bas et je serais restée avec grand plaisir mais... on a préféré rentrer e ton est rentrés. ${ }^{98}$

83 S'il y a certains points communs entre ces migrantes et Maria Casarès - leur origine géographique, la Galice, leur âge au moment de l'émigration (très jeunes adolescentes) - la différence réside néanmoins dans leur origine sociale et leur statut dans la société, au début comme à la fin. Les circonstances de leur apprentissage linguistique en est affecté, leurs bilans de l'expérience migratoire sont variables, autant que leurs personnalités sont variées.

Si Julia raconte de son apprentissage du français qu'elle faisait feu de tout bois, lisait journaux ou revues qui lui tombaient sous les yeux, munie d'un dictionnaire et d'une furieuse envie de sortir d'un statut social misérable, ou la bienveillance d'une patronne qui tenait aux progrès de l'expression de son employée de maison en français ${ }^{99}$, elle doit son expérience linguistique valorisante à la nécessité cruciale d'entrer dans la langue du pays d'accueil, mais surtout à l'acquisition, par sa force de travail, d'une situation bien supérieure à son statut initial et à un ascenseur social en plein fonctionnement durant les Trente Glorieuses. Ses sœurs et son frère, restés en Catalogne, réaliseront un parcours matériellement assez semblable mais les acquis linguistiques et culturels sont incomparables. Le cas de Justa est paradigmatique de l'émigration massive des Galiciens dans les années 1960 à 1980 vers les pays développés d'Europe. Leur émigration est indéniablement une expérience économiquement profitable, leur statut social étant plus élevé, mais leur bilan humain et linguistique est souvent plus nuancé.

Ces expériences vitales, de l'exil politique ou de l'émigration économique, montrent une difficulté variable pour entrer dans la langue étrangère, liée à la classe sociale à laquelle chacune appartiennent : celle pour qui l'enjeu est de conquérir et de maîtriser la langue dans l'objectif élevé de la perfection théâtrale, Maria Casarès, et Julia qui met un point d'honneur tout au long de son parcours vital à acquérir réellement la langue. Pour toutes, l'apprentissage d'une langue a représenté un gain dans leur devenir personnel, que ce soit dans le milieu ouvrier ou dans la carrière d'une actrice exceptionnelle. Sans doute pourrait-on imaginer pour Maria une destinée comparable en Galice dans un milieu social préservé. Mais elle-même était persuadée qu'elle devait sa rencontre avec le théâtre, sa " deuxième naissance » à un effet de "réveil » de la rupture avec son pays et avec sa langue d'origine.

Enfin, observons que l'acquisition de la langue et l'intégration à la société catalane ont été facilitées par un usage, même basique, de la langue castillane comme langue véhiculaire. Ce bilinguisme initial leur a servi à mieux assimiler une troisième langue, catalan, français (Julia) ou italien (Justa), langues qui reposent sur un substrat commun. La plupart sont devenues trilingues mais c'est le galicien qui tend à se perdre dans la migration, dès la $2^{\mathrm{e}}$ génération à $80 \%$, totalement à la $3^{\mathrm{e}}$. La disparition de la pratique 
sociale du galicien tend à le faire disparaître, substitué par la langue du pays d'accueil dont la notion disparait aussi. Mais le galicien laisse-t-il des traces dans ces langues en contact dans la migration?

\section{Des traces de la migration dans les langues, ce que le galicien fait au catalan et à l'espagnol}

\section{Entre galicien et catalan, une affaire de migrations}

En réalité, castillan et catalan ne laissent percevoir que d'infimes traces de galicien, et nous pourrions évoquer le concept du presque-rien forgé par le philosophe Vladimir Jankélévitch pris dans la difficulté de définir la philosophie qui tout entière réside dans ce presque rien. Un champ d'étude exigu de la linguistique leur faisant une place, notamment développée par un spécialiste des "dialectes del català", Joan Veny, dont nous utiliserons notamment deux articles ${ }^{100}$ consacrés aux interférences lexicales entre le galicien et le catalan :

Entre des langues voisines, il est logique qu'il y ait une osmose linguistique. Mais les langues éloignées comme le sont le galicien et le catalan, elles se prêtent moins à ce jeu d'emprunts lexicaux. ${ }^{101}$

L'« osmose linguistique [est] logique entre langues voisines", mais le galicien et le catalan étant des "langues éloignées", elles "se prêtent moins» au «jeu des emprunts ». Elles ne s'influencent pas au-delà du lexique, et « l'importation de mots de Galice en Catalogne s'est souvent réalisée à travers le castillan ", langue véhiculaire pour les Espagnols entre eux. Cependant, « les contacts humains n'ont pas manqué entre les deux communautés ». Nous l'avons vu, après la guerre « une vague dense d'immigrés galiciens a été enregistrée en Catalogne» qualifiée ici d'"avalanche»; on disait couramment que «la ville la plus grande de Galice était Barcelone». Les influences dépendent en partie des politiques linguistiques et «il ne faut pas oublier l'officialité exclusive du castillan ».

\section{"Galleguismes ${ }^{102}$ » du catalan}

On trouve des traces nombreuses dans le bottin de Barcelone, la « densité onomastique transmise : Pombo, Pombar, Carballo, Carballeira, Páez, Pais, Diz, etc.» dénotant l'origine galicienne. Certains de ces galicianismes ont été transmis à différentes époques dans des domaines propices comme la gastronomie, la culture ou l'activité économique, et nous commencerons par le symbolique, la vieira (gal. et cast.), qui a supposément "été diffusé à travers la gastronomie galicienne »; il s'agit d'un grand mollusque, la vieira, et de sa coquille, la venera, devenue le symbole de Saint-Jacques-deCompostelle et du célèbre pèlerinage chrétien dont elle la capitale galicienne est l'aboutissement. Les activités économiques sont aussi des occasions d'échanges linguistiques : le peuplier, xop (cat.), chopo (cast.) vient de xop aussi en galicien. Il est signalé comme potentiellement " arrivé d'Aragon à travers le transport du bois réalisé sur les cours fluviaux ». La pêche est la principale activité économique de la Galice, et les Catalans n'en sont pas absents; elle emprunte et prête son lexique : la traíña (gal.) est une "barque de pêche d'encerclement", devenue en catalan la traïnya "par attraction de ce mot plus transparent qui désignait avant un autre type de matériel de pêche». 
allec (cat.) (ou gallego, galicien), le gentilé, a connu une certaine fortune. En Valencien il en est venu à signifier bastaix, camàlic, le portefaix, et gallega, métaphoriquement, [...] la poutre, qui soutient le plafond. Gallega, "en divers endroits de la Catalogne [...] désignait [aussi] une sorte de capote à capuche utilisée par les paysans, les marins, [...] » ou encore « une boîte à tabac à priser, en forme de calebasse de marchand de vin, sans doute en lien avec la cucurbitacée qui faisait partie de l'habit du pèlerin de Compostelle».

91 Dernier exemple plus savant, « la plante nommée galega (du grec "gala", "lait", car on considère qu'elle augmente la sécrétion lactique des vaches) s'est convertie en gallega par l'attraction paronymique du gentilé ».

\section{«Catalanismes » du galicien}

En sens inverse, les Galiciens de retour au pays ont rapporté une langue " contaminée » : vocables anciens et importés, à travers le castillan, accueillis ensuite dans le dictionnaire normatif comme vinagre, grua, correo, reló (du catalan ancien relotge), faena (la tâche) ou le palindrome capicúa (initialement la pièce de domino double), ou enfin, la paella, plat valencien, formé sur le nom catalan de l'ustensile de cuisine qui, en galicien, se dit tixola, la poêle.

Là encore, le lexique spécialisé de la pêche et de la navigation fournit nombre de transferts. La Catalogne développe une importante activité maritime et à partir du XVIII ${ }^{e}$ siècle, pêcheurs et industriels des salaison catalans s'installent sur le littoral galicien, présence reflétée dans l'onomastique galicienne: Garriga, Batista, Sastre, Fàbregas, Domènech, Puig, Baldrich, Borrell, Massó, etc. et contrepartie des lignages galiciens de Catalogne. Les Catalans ont introduit certains arts de la pêche comme la xávega et le bou, techniques et bateaux de pêche à la traîne qui réussissent des prises plus importantes que les arts traditionnels tels que le xeito ou la rapeta (filet statique). Ces techniques, artes del bou ou bou catalán, sont déprédatrices et interdites comme telles jusqu'au xvIII ${ }^{e}$ siècle. Mais l'exploitation piscicole restant aux mains de Catalans, cette "colonisation » va promouvoir l'industrialisation de la pêche. On retrouve les traces de ces contacts: sotavento pour le sens du vent (sous le vent), avante pour adiante, ou rifar qui signifie rompre les voiles par l'action du vent. Enfin certains noms de poissons sont «marqués du sceau du catalan » car ils n'ont pas d'équivalent castillan et sont certainement dus à des contacts directs : virote, pour verat en valencien, le maquereau ; lliberna, (milano en castillan), le grondin.

Les galleguismes du catalan et les catalanismes du galicien sont des traces d'échanges humains, sociolinguistiques, souvent passés par le castillan et reliés à ce que Joan Veny désigne comme « l'aventure migratoire ${ }^{103}$ quand ce n'est pas au secteur économique.

\section{Contacts du galicien et du castillan, une superbe ignorance}

Il y a peu de galleguismos en espagnol (castillan) et certains sont difficiles à discerner des emprunts au portugais. L'influence du castillan est infiniment plus forte sur le galicien, au point que sans des politiques linguistiques volontaristes, les Galiciens ont $\mathrm{pu}$ craindre une substitution progressive de leur langue. Une brève recension de mots venus du galicien en castillan ${ }^{104}$ nous fait retrouver des mots de ces mêmes domaines propices aux échanges et à la diffusion, la gastronomie, quelques-uns de la vie 
courante, culturelle ou religieuse, comme la célèbre Vieira, empruntée par le catalan aussi, mollusque commun des mers de Galice. Le domaine culinaire donne lieu à d'autres emprunts typiques comme l'Albariño, ce célèbre vin blanc fruité, élevé en Galice et dégusté dans toute l'Espagne et au-delà, composé du galicien albar, blanc, (du latin albus, "blanc» associé à une racine indo-européenne *albho- "blanc») et du suffixe le plus répandu -iño, diminutif ou de relation. La filloa de filloa (gal.), du latin foliŏla, 'petites feuilles', est une crêpe fine consommée dans les terres en Galice ; de même le grelo désigne les fanes tendres et comestibles du navet, typique de la gastronomie galicienne ; la Queimada, est une boisson traditionnelle préparée en flambant l'eau-devie (orujo) mélangée à du citron et du sucre.

La culture galicienne exporte ses symboles, le Botafumeiro, le grand encensoir de la cathédrale de Saint-Jacques, est composé du verbe botar 'lancer', 'répandre', du nom fume, la 'fumée' et du suffixe dérivatif -eiro; il signifie 'encensoir' en première acception et, en langue familière, la 'flatterie'. La célèbre morriña, chantée par Rosalía de Castro et tant d'autres emigrantes Galiciens, vient du galaïco-portugais et désigne un sentiment de tristesse mélancolique, nostalgique, en particulier de la Galice natale. Le Sarao, une fête nocturne avec danse et musique, combine les influences galaïcoportugaise, sarão, et latine, seranum, «l'après-midi».

De la vie quotidienne, on retrouve quelques mots comme la chamiza, dérivé du galaïcoportugais chamiça, qui désigne du petit bois servant à allumer le feu; au masculin, chamizo est un arbre ou un morceau de bois à moitié brûlé, chamuscado. D'origine plus lointaine, le chapapote vient du nahuatl, langue vernaculaire des Aztèques au Mexique où il désignait une huile fumante et parfumée ; rapporté par les colons en Cantabrie et en Galice ce terme est un synonyme du castillan alquitrán, lequel est une trace de l'arabe qui désigne le goudron. On trouve encore le calque transparent fayado de faiado (gal.) qui désigne un grenier non habitable. Enfin la vigía est un terme probablement venu du domaine de la navigation militaire, commun avec la Catalogne, et passé du latin au portugais, qui désigne la tour de guet, l'action de surveiller à distance ou la personne qui surveille depuis la tour de vigie; par analogie, il désignera un écueil dépassant de la surface de la mer, comme les côtes de Galice en recèlent tant.

\section{Conclusion}

Le contact des langues dans la contrainte de l'apprentissage d'une langue en situation d'exil ou de migration entraîne des changements, la nature des langues étant dans le contact et le changement. Les migrations font une sorte de "mise à jour " constante non seulement des langues des pays d'accueil, mais également des langues d'origine, produisant des interférences mutuelles. Les migrants enrichissent de mots et de tournures nouvelles les langues autochtones, en fonction de leurs connaissances qu'elles soient savantes ou populaires.

99 Parmi les facteurs facilitant l'acquisition et la maîtrise des langues par les migrants et exilés figure en bonne place le temps passé à parler la langue acquise (langue B). Mais certaines locutrices ou locuteurs gardent plus ou moins longtemps un « accent » ou une tendance à contaminer la langue acquise avec des éléments de la langue initiale (A) tandis que d'autres les effacent. Un second facteur serait leur niveau culturel de départ, quel que soit ce niveau, les accents s'accrochent, les traces persistent bien que le niveau de langue acquis reste influencé par le niveau d'instruction en général. Ainsi, les 
méthodes d'apprentissage jouent certainement un rôle important. Les politiques linguistiques, enfin, sont le conditionnant majeur de la subsistance ou de la disparition des langues. En fonction de leur organisation politique, dominatrices ou tolérantes, volontaristes ou liberticides, elles permettent de minorer des langues, de les maintenir, de les sauver ou de les faire disparaître.

Du point de vue des personnes, nous avons montré que les migrants ne perdent ni le castillan, ni le catalan, ni le galicien dans la migration, qu'ils continuent à les pratiquer leur vie durant.

Maria Casarès était parfaitement bilingue en français et en espagnol (castillan) bien qu'elle ait toujours conservé un léger accent, un deje en espagnol, une trace de son origine. Elle n'a pas eu de descendance. Au contraire, Montserrat n'a jamais réussi à acquérir un usage total de la langue du pays de l'exil. Le retour impossible et l'intégration jamais acquise sont caractéristiques de son groupe social, celui des exilés politiques qui restent souvent dans un entre-deux, entre deux pays, entre deux cultures, entre deux langues, en créant d'autres, celles de l'entre-deux, de l'hybridité décrite par les sociolinguistes. L'un d'eux souligne combien l'interlangue des migrants témoigne moins de leur difficulté d'adaptation que de l'effort inimaginable qu'ils produisent pour s'adapter, inconcevable pour qui n'a pas fait l'expérience du déplacement. La langue des guérilleros antifranquistes, comme celle de Montserrat, ne s'adapte que dans une résistance forte, signe d'une lutte vitale pour la reconnaissance.

Dans la migration, le galicien s'est affaibli le premier et il en reste peu de traces dans la langue catalane, quelques-unes dans la langue dominante espagnole (castillan). Mais le catalan aussi se perd, pour la deuxième génération des migrants à l'étranger sous des influences et des contraintes linguistiques plus fortes. L'allemand, le français, deviennent les langues propres pour la deuxième génération et supplantent l'usage des deux (voire trois) langues vernaculaires. Seul le castillan, ici espagnol, langue véhiculaire dans la famille, survit à grand-peine pour une génération et subit le sort des langues dominées du parcours migratoire, il disparait à la génération suivante. Dans la vieillesse et atteinte d'un syndrome dit d'Alzheimer, c'est le français qui a disparu le premier pour Julia, mais ses trois autres langues apparaissant de manière aléatoire, comme dans un pidgin, sur la structure de celle qui les domine toutes, le castillan. Ses enfants conservent l'usage d'un castillan affaibli, teinté de français ou réappris. Le galicien et le catalan de leur enfance ont été soit réappris volontairement soit perdus. La transmission s'arrête à la $2^{e}$ génération y compris pour le castillan, perdu à $50 \%$ dans la $3^{\mathrm{e}}$ dans la migration à l'étranger. L'ensemble de la fratrie restée en Catalogne est devenue parfaitement trilingue, passant selon les besoins de la communication du catalan au castillan et plus exceptionnellement, au galicien. Leurs enfants sont tous bilingues, catalanophones - voire catalanistes - et castillanophones dans une Catalogne où la question de la diglossie se pose à nouveau en termes virulents et semble éloigner un possible bilinguisme équilibré pourtant souhaité par une partie de la population et rêvé par la communauté des sociolinguistes natifs. Les enfants de la deuxième génération ont fréquenté une école catalane où l'enseignement en castillan dominait,

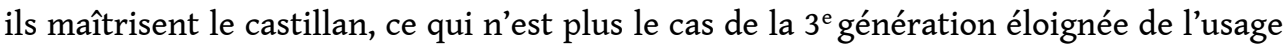
$\mathrm{du}$ castillan par une politique linguistique volontariste et efficace de sauvegarde du catalan.

Perte ou gain? L'évolution de ces trois langues d'Espagne, dominantes ou menacées de disparition est en cours, encore incertaine. Une nouvelle page semble vouloir se 
tourner et dans cet état transitoire, des propositions émergent d'une Espagne plurilingue et multiculturelle.

104 La question des politiques linguistiques, cruciales dans l'évolution des langues, restera à aborder. "L'écologie des langues ", champ de recherches émergé dans les années 1970, observe les langues dans leur environnement social, les relations entre elles et ceux qui les parlent et même les relations avec la géographie d'un lieu ${ }^{105}$. Soutenant la diversité linguistique et défendant les langues «minorées» ou "menacées de disparition ", cette approche "un peu darwinienne " selon les auteurs eux-mêmes, considère les langues comme des espèces ${ }^{106}$, observe leur évolution et lutte contre leur disparition. Les auteurs parlent à ce titre d' "évolution linguistique " ou d' " écosystèmes linguistiques », faisant reculer l'horizon des possibles linguistiques et, pourquoi pas, permettant d'espérer un élargissement du champ des possibles politiques.

105 Gageons que cette voie qui s'ouvre avec l'impérieuse nécessité de la défense et du maintien de la diversité sous tous ses aspects, ne sera pas un chemin parsemé que de roses.

\section{NOTES}

1. Odette Martinez-Maler, fille de Francisco Martinez-Lopez, dit "El Quico", rapporte que les derniers guérilleros actifs ou cachés en Galice, sont "O Piloto" jusqu'en 1963 et "O Pinche" jusqu'en 1968.

2. Chiffres établis par Guy Hermet, Les Espagnols en France, Paris, éditions Ouvrières, 1967 et Javier Rubio, La emigración española a Francia, Barcelona, Ariel, 1974.

3. Brigitte Lestrade, «L'immigration espagnole en France et en Allemagne de 1960 à 1973 : un modèle d'intégration?», in Serge Gouazé, Anne Salles et Cécile Prat Erkert (dir.), Les enjeux démographiques en France et en Allemagne: réalités et conséquences, Villeneuve d'Ascq, Presses Universitaires du Septentrion, 2011, p. 209-234, https://books.openedition.org/septentrion/ 16045\#authors, consulté le 13 juillet 2019.

4. Ibid.

5. Ophélia Avron, L'émotion, source de connaissance, Paris, L'Harmattan, 2017, p. 13.

6. Ibid. p. 17.

7. Ferdinand de Saussure, Cours de linguistique générale, [1916], texte établi par Charles Bally, Albert Sechehaye et Albert Riedlinger, Paris, Payot, 1971, p. 25.

8. Claude Raffestin, «La langue comme ressource, pour une analyse économique des langues vernaculaires et véhiculaires" Cahiers de géographie du Québec 2256, 1978, p. 281. https:// www.erudit.org/fr/revues/cgq/1978-v22-n56-cgq2629/021396ar.pdf, consulté le $1^{\text {er }}$ août 2019.

9. Emprunt, en français, préstamo (prêt) en espagnol, loan ou borrowed word (emprunt) en anglais (nous précisons).

10. Marius Sala, Ibid. Pour plus d'informations sur le débat sur le substrat Marius Sala renvoie aux travaux de Bertil Malmberg, notamment à son ouvrage Les nouvelles tendances de la linguistique, Paris, PUF, 1966. 
11. Eugenio Coseriu, Sincronía, Diacronía e Historia, El problema del cambio linguistico, Madrid, Gredos, 1973, p. 283.

12. André Martinet, Économie des changements phonétiques. Traité de phonologie diachronique, ésitions Maisonneuve et Larose, Paris, 2005, p. 24 et p. 71 citant George Kingsley Zipf, Human Behaviour and the Principal of Least Effort, Cambridge Mass, 1949.

13. Coseriu, op. cit. p. 11, c'est le titre du premier chapitre de l'ouvrage.

14. Martinet, op. cit.

15. Salikoko S. Mufwene, The Ecology of Language Evolution, Cambridge University Press, 2001, p. 138

16. Marius Sala, op. cit., p. 11-12. « [...] el bilingüismo es un fenómeno que afecta la mayoría de las poblaciones de la tierra. [...] La actual visión de los lingüistas de considerar el unilingüismo como regla y el bilingüismo o plurilingüismo como fenómeno excepcional, no concuerda con la realidad [...][en la que] para satisfacer las necesidades de comunicación, la mayoría de los hablantes de la tierra conocen y usan más de un idioma. ». (Nous traduisons, comme dans l'ensemble de l'article nous avons traduit les citations en langue étrangère).

17. Henri Boyer, op. cit. p. 65.

18. Ibid.p. 87.

19. Source : www.touteleurope.eu/actualite/les-langues-officielles-de-l-union-europeenne.html, consulté le 24 juillet 2019.

20. Pierre Encrevé, en réponse à la question: "Quelles sont les conséquences d'une reconnaissance légale [des langues de France dans la Charte européenne des langues régionales ou minoritaires]?", in Jérôme Bouvier et al. (dir.), «II. LaCharte: conceptions et perspective française ", Langues régionales : langues de France, langues d'Europe, Paris, Éditions de la Bibliothèque publique d'information, 2003, p.14-30, http://books.openedition.org/bibpompidou/1477, consulté le 24 juillet 2019.

21. Marius Sala, "Lenguas en contacto en el ámbito hispánico", Actas del XII Congreso de la AIH (Asociación Internacional de Hispanistas), Instituto Cervantes, 1995, p. 28, disponible sur https:// cvc.cervantes.es/literatura/aih/aih_xii.htm, consulté le 19 juillet 2019: «es importantísima porque supone investigar la evolución de la lengua en función del desarrollo sociolingüístico de la población que la habla ».

22. Iorgu Iordan, « Du bilinguisme dans le domaine roman » in Actes du XIII ${ }^{e}$ congrès international de linguistique et de philologie romanes, Presses de l'Université de Laval, 1976, t. I, p. 5-15.

23. Jakobson, de même, évoque l'exemple des "influences réciproques " entre le polonais dominant aux $\mathrm{XV}^{\mathrm{e}}$ et $\mathrm{XVI}^{\mathrm{e}}$ siècles sur ses voisins de l'est qui s'est pourtant adapté au biélorusse et à l'ukrainien tout en conservant son statut de langue dominante par le fait qu'il restait la langue officielle. C'est le polonais qui s'est nuancé d'emprunts aux langues parlées et utilisées dans les territoires politiquement dominés.

24. Denise Jodelet donne la définition suivante des représentations sociales : «C'est une forme de connaissance socialement élaborée et partagée, ayant une visée pratique et concourant à la construction d'une réalité commune à un ensemble social.", et plus loin, comme un "savoir "pratique" [qui] sert à agir sur le monde et sur autrui ». Denise Jodelet (dir.), Les représentations sociales, Paris, PUF, 1999, p. 53 et 61.

25. Henri Boyer, op. cit., p. 62.

26. Denise Jodelet, op. cit., p. 53.

27. Antoine Meillet pour le français ou Marius Sala pour l'espagnol par exemple.

28. Uriel Weinreich, Languages in contact, Paris, The Hague, Mouton \& Co., 1970, p. 64-67.

29. Ibid., p. 66-67

30. Uriel Weinreich, "the bilingual speaker is the ultimate locus of language contact", Languages in contact. Paris, The Hague, Mouton \& Co., 1970, p. 71.

31. Uriel Weinreich, Languages, op. cit. 
32. Henri Boyer, Introduction à la sociolinguistique, Paris, Dunod, 2017, p. 115.

33. Henri Boyer, dans " "Diglossie": un concept à l'épreuve du terrain. L'élaboration d'une sociolinguistique du conflit en domaines catalan et occitan", Lengas, revue de sociolinguistique, $\mathrm{n}^{\circ}$ 20, Les espaces de la diglossie, Montpellier, U.A. 04 1053, 1986, p. 21-54, cite R. Lafont, "Pour retrousser la diglossie ", Lengas $n^{\circ} 15$, p. 7 et «Le discours idéologique dans l'enquête sur la diglossie $»$, Cahiers de la linguistique sociale $\mathrm{n}^{\circ} 4-5, \mathrm{p} .92$.

34. Ibid.

35. Rodolfo Gutiérrez, établit à partir des recensements de l'INE (Instituto Nacional de Estadística) une constante augmentation, entre 2000 et 2006, des communautés linguistiques immigrantes parlant une autre langue que l'espagnol. Rodolfo Gutiérrez, "Lengua, migraciones y mercado de trabajo", édité par Fundación Telefónica y el Instituto Complutense de Estudios Internacionales, El valor económico del español: una empresa multinacional, $\mathrm{n}^{\circ}$ DT 05/07, s.d. https://core.ac.uk/ download/pdf/6228941.pdf, consulté le 7 avril 2020.

36. F. Xavier Vila, “¿Quién habla hoy en día castellano en Cataluña? Una aproximación demolingüística", in Dolors Poch Olivé (dir.), El español en contacto con las otras lenguas peninsulares, Madrid, Iberoamericana-Vervuert, 2016, p. 135-155.

37. Emma Rubio, «Un exemple de "transculturation" de basse intensité: l'immigration galicienne en Catalogne", in Les Langues néolatines: revue de langues vivantes romanes, Société des Langues Néolatines, Association des Enseignants de Langues Vivantes Romanes, 2015, "Migrations et transculturation, fin du Xx et début du XXI ${ }^{\mathrm{e}}$ siècles ", vol. I, n 374 , p. 89, http:// www.neolatines.com, ou HAL : hal-01366519, consulté le 12 juillet 2019. p. 89.

38. Henri Boyer, op. cit. p. 25.

39. Georges Steiner, After Babel, New York and London, Oxford University Press, 1975, p. 75, cité par Claude Raffestin, op. cit., p. 281.

40. Une infinité des réalisations sont observables pour les locuteurs et dans les langues, la variation étant le «trait constitutif majeur des langues historiques : la diversité est inscrite dans leur usage social. [...] [0]n s'accorde à repérer (au moins) cinq types de variations linguistiques au sein d'une même communauté [...] L'origine géographique [...], L'origine sociale, l'appartenance à un milieu socioculturel ", "L'âge.", «Les circonstances de l'acte de communication ", "Le sexe », in Henri Boyer, Introduction, op. cit., p. 33-48.

41. Abdelmalek Sayad, La double absence : des illusions de l'émigré aux souffrances de l'immigré, Paris, Seuil, 1999.

42. Pierre Bourdieu, Ce que parler veut dire. L'économie des échanges linguistiques, Paris, Fayard, 1982.

43. Boyer, Introduction, op. cit., p. 24.

44. Weinreich, op. cit., p. 71.

45. Entretiens que nous avons réalisés en 2014 et 2016 en Galice, en Catalogne et en France dans le cadre de recherche sur les migrations intérieures dans les années 1940-1960.

46. Lydie Salvayre, Pas pleurer, Paris, Éditions du Seuil, 2014.

47. Benoît Filhol et Maria del Mar Jiménez Cervantes-Arnao, “El frañol en Pas pleurer de Lydie Salvayre y su traducción al español”, Çédille, revista de estudios franceses, n 14 , avril 2018, p. 197-220. On pourra se référer à cet article pour la précision des décomptes systématiques d'occurrences en fragnol, leur analyse de l'usage qu'en fait l'auteure. Cet article a largement inspiré notre réflexion et nous en formulerons des éléments synthétisés. Il a souvent confirmé nos propres relevés de début de recherche.

48. Filhol et Jiménez, Ibid., p. 199.

49. Filhol et Jiménez citent Lydie Salvayre, Ibid., p. 200.

50. Langue hybride utilisée par les immigrés dans leur nouveau lieu de résidence. Un pidgin se caractérise par l'emploi de structures formelles de la langue d'origine complétées avec des mots de la langue nouvelle. Gabriel Mannessy, en 1995, le différencie du «créole (mot d'origine espagnole qui a désigné primitivement les individus impliqués, dominants aussi bien que 
dominés, dans la colonisation) [qui] est un vernaculaire, c'est-à-dire une langue maternelle, première, d'une communauté plus ou moins importante.", Gabriel Manessy, Créoles, pidgins, variétés vernaculaires. Procès et genèse, Paris, éditions du CNRS, 1995, p. 27.

51. Salvayre, op. cit., p. 16.

52. "se organizaban grandes comidas, se contaban chistes verdes y nadie se compraba muebles porque todo el mundo creía que terminaría volviendo a la patria abandonada", Salvayre, ibid.

53. Gabriel Manessy, Créoles... op. cit.

54. Filhol et Jiménez, op. cit., p. 200.

55. Ibid., p. 201.

56. Lydie Salvayre, No llorar, Madrid, Anagrama, 2015. Dans la conclusion de l'article dont la deuxième partie est consacrée à l'édition du roman en espagnol et à la difficulté de traduire la langue hybride, Filhol et Jiménez saluent la traduction: "no se aleja demasiado de la obra original", mais pour le fragnol, "componente clave para la obra y el personaje de Montse", elle échouerait: "creemos que se le resta sentido a la obra", autrement dit la réception s'en trouve amputée pour les hispanophones: "dejando de lado el juego lingüístico, elemento de gran importancia en Pas pleurer". La gageure de transmettre dans la langue du pays d'origine cette dimension linguistique particulière de l'exil, qui engage l'identité des personnages et l'expérience de l'exilée reste un défi.

57. Ibid. p. 82. Henri Boyer parle de l'« inquiétude» des migrants de parler correctement qui leur fait commettre des erreurs par souci d'hypercorrection. C'est bien le cas de Montserrat « qui s'est évertuée, depuis son arrivée en France, à corriger son accent espagnol, à parler un langage châtié et à soigner sa mise pour être toujours plus conforme à ce qu'elle pensait être le modèle français (se signalant par là même par sa trop stricte conformité, comme une étrangère) ».

58. Ibid. p. 279.

59. Filhol et Jiménez, op. cit., p. 202.

60. Ibid., p. 202-203.

61. Christian Lagarde, Le parler « melandjao » des immigrés de langue espagnole en Roussillon, Presses universitaires de Perpignan, 1996, p. 251.

62. Ibid., p. 8.

63. Ibid., p. 9.

64. Maria Casarès, Résidente privilégiée, Paris, Arthème Fayard, 1980 : «mon nom qu'il m'a fallu affubler d'un accent grave pour lui restituer le $s$ de la fin », p. 209.

65. Albert Camus et Maria Casarès, Correspondance 1944-1959, Paris, Gallimard, 2017. La publication de cette correspondance à l'initiative de la fille de l'écrivain, Catherine Camus, a redonné à la célèbre comédienne une grande actualité médiatique.

66. Casarès, op. cit.

67. Ibid., p. 202

68. Ibid., p. 201-202.

69. Ibid., p. 204.

70. Ibid., p. 202.

71. Entretien avec Maria Casarès, mené par Moussa Abadi, Producteur RTF (Radiodiffusion Télévision Française) Paris, 1961, (6 min. 30), source Ina: www.ina.fr/audio/PHD89009987/ entretien-avec-maria-casares-audio.html, consulté le 11 juillet 2019.

72. Casarès, op. cit., p. 203.

73. Francisco Martinez-Lopez, dit "El Quico", Guérillero contre Franco, la guérilla antifranquiste du León (1936-1951), Paris, Syllepse, 2000, p. 122.

74. Ibid., p. 126-127.

75. Ibid., p. 138.

76. Claude Raffestin, op. cit. p. 280, cite Henri Gobard en 1966 (L'analyse linguistique, analyse tétraglossique, Paris, Flammarion) qui classe les langues véhiculaires et les définit comme 
langage(s) «national ou régional, appris par nécessité, destiné aux communications à l'échelle des villes ». C'est bien ce rôle que joue le castillan entre les migrants galiciens et les Catalans.

77. Montes Lopez María, "Le galicien ", La linguistique, vol. 44, 2008/1, p. 29-40, https:// www.cairn.info/revue-la-linguistique-2008-1-page-29.htm, consulté le $1^{\text {er }}$ août 2019, p. 37-38.

78. Le terme de "bonne" a, en français, une consonance péjorative. Nous le reprenons volontairement puisque c'est ainsi qu'elles étaient nommées : las "minyonas" en catalan, las "criadas" en castilla, les «bonnes » espagnoles en français. Bruno Tur, «Españolas en París ou la solitude des "bonnes à tout faire" espagnoles à Paris ", Décadrages, n 14, 2009, http:// journals.openedition.org/decadrages/332, consulté le 4 avril 2020 ; Laura Oso Casas, notamment : «Bonnes et concierges espagnoles à Paris : immigration et rapports de domination de classe dans le secteur professionnel du service domestique ", trad. Vicente Lillo Marqués et Nicole Lillo Marqués, Exils et migrations ibériques au $\mathrm{xX}^{e}$ siècle, revue du CERMI, 2006, https://www.persee.fr/ doc/emixx_1245-2300_2006_num_3_2_1087, consulté le 4 avril 2020.

79. "Mira llegué de Fruxil a Barcelona, en Fruxil era el gallego, en Barcelona era el catalán. Yo me encontré igual cuando llegué a Cataluña que cuando llegué a Francia: no sabía una palabra!” : Julia, $4^{\mathrm{e}}$ partie, entretiens réalisés en 2014 et 2016 à Fruxil (Lugo, Galice) et à Paris.

80. "Para mí no fue trauma ninguno. Me contestaban en español o me decían japrende el catalán! Porque los catalanes eran así. Pero yo a los tres meses ya hablaba el catalán." : Julia, ibid.

81. "A mí nunca me lo han prohibido... Yo no me acuerdo de que estuviera prohibido": Julia, ibid. 82. "Yo hablaba castellano porque yo no sabía hablar otra cosa. Menos mal que yo hablaba el castellano. Yo creo que en Galicia - y en Cataluña debe ser igual - no querían el español pero en Galicia... ¡yo bien había aprendido el español! / Q.: Y el gallego lo hablabas... / ¿En Galicia sí!”, Julia, ibid.

83. "Yo me integré muy bien. No sé por qué yo no me sentí extraña en ningún sitio." : Julia, ibid.

84. Vull dir (cat.), quiero decir (cast.)

85. “ Cuando llegué he pensao: tú no entiendes nunca nada desto... Pero [...] estuve yo siempre, desde que llegué, con catalanes. Y así poco a poco fui cogiendo la lengua, a mi manera, como me salía porque hay quien le da vergüenza de hablar catalán y hablarlo mal. Prefiere no hablarlo... Pero ¡yo no! Yo lo hablaba, y aún ahora, a lo mejor en medio digo una [palabra] castellana, pero “ vull dir" que no me ha hecho daño eso.", Luisa, entretien du 20 août 2014 à Manresa, en Catalogne.

86. Nanos (cat.) niños (cast.).

87. “Con que estás [...] sirviendo siempre estás con ellos y ya vas cogiendo una [palabra], vas cogiendo otra... Yo que tenía allá tres nanos, tres niños que uno era pequeñito que lo (sic) tenía de [...] llevarlo al colegio [...] y el nano ¡ igual que si [yo] fuera de la casa ! Y también aprendes ¡ con que hablan siempre!", Luisa, ibid.

88. "en medio de la gente siempre hay alguien que a lo mejor habla mal de los emigrantes, pero yo no, no me he encotrao". Ibid.

89. Charnego: terme péjoratif utilisé en Catalogne pour désigner l'«immigré qui vient d'une région de l'Espagne où l'on ne parle pas le catalan », Diccionario de la lengua española, https:// dle.rae.es, consulté le 11 août 2019. À l'origine le mot désignait dans le sud de la France un «bâtard» né d'un Français et d'une Catalane. Bien que les Andalous ou les Extrémègnes aient davantage souffert de cette stigmatisation, les Galiciens, souvent méprisés par les autres Espagnols pour leur ruralité et leur pauvreté, en ont également été affublés lors des vagues migratoires internes.

90. "nos decían los charnegos a los que veníamos de fuera, ¡los charnegos!", "aquí éramos españoles pero deso, charnegos...", Maria Luz, entretien fin août 2014.

91. "Algunos [te decían] ¡háblame en cristiano! si se les escapaba [algo en gallego] claro... es que hay gente que puede hablar el castellano, pero hay otros que no porque la gente que son de pueblo... muy cerraos y tal y van a la policía... Eran unos matones sats...", ibid. 
92. «Bien sûr qu'on le parlait! ", ibid.

93. Barreja (cat.), mezcla (cast.), le (fameux) « mélange».

94. "Si te digo que ir a Galicia para dos meses, irme allí donde nací una temporadita, a Fruxil, estar dos o tres meses sí, pero para la vida estoy más adaptada aquí. Irme decir ahora vives en Sarria eso no, no lo haría. Lo que hoy te ilusiona mañana ya no... pierdes mucha ilusión...", Maria Luz, ibid.

95. "la niña quedaba con nueve meses y medio... aún estaba dando el pecho... ¡Mira qué contenta iría yo para allá! ... Adelgacé unos diez quiilos... quiero mejor no acordarme... eso ya pasó...", Justa, entretien réalisé chez elle le 3 octobre 2014, à Rubián de Cima (près de Cervela, Lugo), en Galice.

96. "El italiano lo he aprendido en seguida porque es muy parecido al gallego. Nada más que lo oyes hablar lo aprendes en seguida. A mí ya me preguntaban si era italiana porque lo hablaba sin estudiarlo.", Justa, ibid.

97. "Fui a unas clases pero lo dejé porque no lo podía comprender. Además dije para qué voy a aprender el alemán si ya no lo voy a hablar... No lo necesitaba ¿para qué? Si yo a Alemania... no iba a ir.", Justa, ibid.

98. "Los gallegos tenemos mucha morriña por nuestro pueblo pero yo me quedaba allí y llevaba la niña para allí y me quedaba de buena gana pero... en fin nosotros como teníamos aquí [también] los padres y los hermanos... Pues nos gustaba más venirnos para aquí, y nos vinimos.", Justa, ibid.

99. Bruno Tur, L'immigration espagnole à Paris dans les années 1960: discours, représentations et stéréotypes, thèse de doctorat, Université Nanterre Paris X, 2014, p. 460-462, montre qu'il était souhaité par « les employeuses » que les «bonnes » espagnoles, connaissent rapidement un peu de français, mais surtout du dernier chic qu'elles-mêmes connaissent un peu d'espagnol car « La "bonne" espagnole a tout à apprendre de son employeuse qui doit l'éduquer ». Voir les manuels publiés à l'époque dont il propose une analyse des stéréotypes, comme celui de Solange Fasquelle, Conchita et vous, Paris, Albin Michel, 1968.

100. Joan Veny, "Galleguismes del català", La Vanguardia, édition du vendredi 31 mars 2000, p. 7, http://hemeroteca.lavanguardia.com/preview/2000/03/31/pagina-7/34037729/pdf.html?

search=galleguismes et "Catalanismes del gallec", La Vanguardia, édition du vendredi 7 avril 2000, p. 5, http://hemeroteca.lavanguardia.com/preview/2000/03/31/pagina-5/34054378/pdf.html? search=galleguismes, consultés le 6 août 2019.

101. "Entre langues voisines il est logique qu'il y ait une osmose linguistique. Mais les langues éloignées, comme le sont le galicien et le catalan se prêtent moins à ce jeu des emprunts lexicaux ». Nous présenterons dans les lignes suivantes les citations traduites. », Jean Veny, ibid. 102. Galleguisme en catalan. Le terme français attesté est celui de galicianisme.

103. Joan Veny, Idem.

104. La plupart des définitions sont issues du dictionnaire de la Real Academia Española: Diccionario de la lengua española, 23. a ed., [versión 23.2 en línea], https://dle.rae.es/, consulté le 8 août 2019; d'autres vérifications ont été effectuées dans d'autres dictionnaires: Breve diccionario etimológico de la lengua castellana, http://etimologias.dechile.net, consulté le 8 août 2019; Real Academia Gallega: https://academia.gal/, consulté le 8 août 2019, Dictionnaire Espagnol-Français, https://www.wordreference.com/esfr/, consulté le 8 août 2019 pour certaines traductions ; TLFI Dictionnaire de la langue française, https://www.cnrtl.fr/definition/, consulté le 6 avril 2020, pour le français.

105. Romain Mielcarek, Écologie linguistique: la langue est un loup pour la langue, rfi, les voix du monde, émission du 24 avril 2015, URL :http://www.rfi.fr/hebdo/20150424-ecologie-linguistiquemort-naissance-langues-lacito-cnrs-unesco/, consulté le 10 juillet 2019.

106. Ibid., citant Jean-Louis Calvet. 


\section{RÉSUMÉS}

Parmi les nombreuses contraintes, difficultés et injonctions d'adaptation auxquelles les migrants sont confrontés se trouve celle de la langue. L'apprentissage d'une langue supplémentaire met les langues en contact. Montrer que la migration laisse des traces y compris dans les langues parlées et apprises par les migrants et comprendre comment les migrants espagnols, exilés politiques ou migrants économiques se sont confrontés à cette question fait l'objet de cet article qui s'inspire des travaux de la sociolinguistique notamment sur les langues en contact. L'Espagne et ses quatre langues historiques devenues co-officielles dans la constitution de 1978 en constitue l'un des laboratoires les plus célèbres.

Entre las numerosas dificultades que se le imponen al migrante en términos de adaptación se encuentra la lingüística. El migrante se enfrenta con la necesidad de aprender un nuevo idioma. La migración por naturaleza pone las lenguas en contacto. Descubrir las huellas de la migración dentro de los sistemas lingüísticos, inicial o adquirido, y entender cómo los migrantes españoles, exiliados políticos o emigrantes "económicos", lidiaron con esta necesidad es el objeto de este artículo inspirado de la sociolingüística, especialmente del contacto de las lenguas. España, con sus cuatro lenguas históricas ya cooficiales en la constitución del 78, constituye un laboratorio famoso para el estudio de dicho contacto.

\section{INDEX}

Mots-clés : langues d'Espagne, migrations, langues en contact, sociolinguistique, histoire sociale Palabras claves : lenguas de España, migraciones, lenguas en contacto, sociolingüística, historia social

\section{AUTEUR}

\section{EMMA RUBIO-MILET}

Laboratoire LISAA, Université Gustave Eiffel (ex UPEM) 